\title{
$e^{ \pm}$-rich GRB Fireball and Infrared Flashes
}

\author{
Zhuo Li, Z. G. Dai, and T. Lu \\ Department of Astronomy, Nanjing University, Nanjing 210093, P. R. \\ China
}

\begin{abstract}
Gamma-ray bursts (GRBs) are believed to originate from ultra-relativistic fireballs, with initial Lorentz factor $\eta \sim 10^{2}-10^{3}$. However very high energy photons may still suffer from $\gamma \gamma$ interaction. We show here that in a wide range of model parameters, the resulting pairs may dominate electrons associated with the fireball baryons. This may provide an explanation for the rarity of prompt optical detections. A rapid response to the GRB trigger at the IR band would detect such a strong flash.
\end{abstract}

\section{Introduction}

GRB990123 is accompanied with a prompt optical flash which is generally interpreted as emission of the electrons accelerated by a reverse shock (Sari \& Piran 1999), which forms when the fireball interact with its surrounding medium. However, strong optical flashes appear to be rare, since they have not been detected from other bursts. We show below that the intense pair production may occur within the fireball in the bursting phase. The presence of a large number of $e^{ \pm}$pairs decreases the shared energy per lepton in the reverse shock so that the reverse flash is softened to the IR band. This may provide an explanation for the rarity of prompt optical detections.

\section{Pair loading and IR flash}

Since during the burst phase, the very high-energy GRB photons could still suffer from $\gamma \gamma \rightarrow e^{ \pm}$absorption, intense pair production may occur within the fireball. The observed photon spectra of GRBs can be approximated by a broken power-law, with a high-energy portion of the form $N_{\epsilon} \propto \epsilon^{-\beta}$ for $\epsilon>\epsilon_{b}$, where $\epsilon=h \nu / m_{e} c^{2}$ is the photon energy in units of the electron's rest energy, $\epsilon_{b} \sim 1$ is the break energy above which the index $\beta \sim 2-3$. A photon with energy $\epsilon$ may annihilate any photons above $\epsilon_{a n}=\eta^{2} / \epsilon$, its optical depth is given by (Lithwick \& Sari 2001) $\tau_{\gamma \gamma}(\epsilon)=(11 / 180) \sigma_{T} N_{>\epsilon_{a n}} /\left(4 \pi R_{\gamma}^{2}\right)$, where $N_{>\epsilon} \equiv \int_{\epsilon}^{\infty} N_{\epsilon} d \epsilon$ and $R_{\gamma}$ is the radius where a GRB takes place. For emission from relativistic expanding spheres $(\eta \gg 1)$ we always have $R_{\gamma}=\eta^{2} c \delta t$, with $\delta t$ the rapid variability timescale in light curves. Setting $\tau_{\gamma \gamma}\left(\epsilon_{c}\right)=1$, each GRB photon above $\epsilon_{c}$ would be absorbed to produce a pair of $e^{ \pm}$. Integrating the photon spectrum above $\epsilon_{c}$, 
we have the total number of the resulting pairs

$$
\begin{aligned}
N_{ \pm} & =N_{>\epsilon_{c}}=\left(\frac{\beta-2}{\beta-1}\right) \frac{E_{\gamma}}{\epsilon_{b} m_{e} c^{2}}\left(\frac{\epsilon_{c}}{\epsilon_{b}}\right)^{-(\beta-1)} \\
& =8 \times 10^{53} \delta t_{-1}^{-2} E_{\gamma, 53}^{2} \epsilon_{b}^{2 \beta-4} \eta_{2.7}^{-2(\beta+1)}
\end{aligned}
$$

and hence the ratio $k_{ \pm} \equiv N_{ \pm} / N_{b}=6 \delta t_{-1}^{-2} \varepsilon E_{\gamma, 53} \epsilon_{b}^{2 \beta-4} \eta_{2.7}^{-(2 \beta+1)}$, where $E_{\gamma}=$ $\int \epsilon m_{e} c^{2} N_{\epsilon} d \epsilon$ is the observed GRB energy, $\varepsilon \equiv E_{\gamma} / E_{k}$ is the GRB efficiency, and the numerical coefficients on the right-hand sides correspond to $\beta=2.2$. Hereinafter, we adopt the convention $Q=10^{x} Q_{x}$ and cgs units. Once $\eta$ less than $\sim 800$, we will have $2 k_{ \pm}>1$ indicating that the fireball becomes pair-rich. Since the ratio of the pair annihilation timescale $t_{a n} \sim\left(k_{ \pm} n_{b} \sigma_{T} c\right)^{-1}$ (with $n_{b}$ the baryonic density in comoving frame) to the expansion time $t_{d} \sim R_{\gamma} / \eta c$ is usually less than unit, pair annihilation can be negligible.

The pairs will be carried by fireball into the revere shock to give rise to a reverse flash. The reverse shock is usually mildly relativistic, the characteristic Lorentz factor (comoving frame) of the shocked leptons (baryonic electrons and pairs) is therefore $\gamma_{m} \simeq \xi_{e} \mu$, where $\xi_{e}$ is the fraction of energy carried by the shocked leptons, $\mu$ is the effective mass per lepton in units of electron mass, i.e.,

$$
\mu m_{e}=m_{p} \frac{1+2 k_{ \pm}\left(m_{e} / m_{p}\right)}{1+2 k_{ \pm}} \simeq \begin{cases}m_{p} & \text { pair - poor } \\ \left(2 k_{ \pm}\right)^{-1} m_{p} & \text { pair - rich }\end{cases}
$$

For $2 k_{ \pm}>1$, the observed peak frequency of synchrotron photons $\nu_{m} \propto \gamma_{m}^{2}$ is lower by a factor of $\left(2 k_{ \pm}\right)^{-2} \sim 7 \times 10^{-3}\left(k_{ \pm} / 6\right)^{-2}$. While a pair-poor reverse shock produces a UV/optical flash (Sari \& Piran 1999), a pair-rich flash will move to $<10^{13} \mathrm{~Hz}$. Because the synchrotron self-absorption is important at $<10^{13} \mathrm{~Hz}$, the emission will peaks at this IR band (Li, Dai \& Lu 2002, submitted).

\section{Discussion}

Since the bulk Lorentz factor in GRB990123 may be quite large, e.g., $\eta \approx 1200$ (Wang, Dai \& Lu 2000), as well as its MeV spectrum is somewhat soft with $\beta \simeq 2.71$ (Briggs et al. 1999), GRB990123 should be in the $2 k_{ \pm}<1$ case (see eq.[1]). We expect IR flashes with $2 k_{ \pm}>1$ are more common in a wide range of model parameters. Note that $N_{e} \simeq N_{ \pm}$for $2 k_{ \pm}>1$, the peak flux of the flash $F_{\nu, \max } \propto N_{e}$ will be increased by a factor of $2 k_{ \pm}$, so the IR flash is strong. Unlike optical emissions, IR emissions will not suffer dust obscuration if GRBs occur in star formation regions.

\section{References}

Briggs, M. S., et al. 1999, ApJ, 524, 82

Lithwick, Y., \& Sari, R. 2001, ApJ, 555, 540

Mészáros , P. 2002, ARA\&A, 40, 137

Sari, R., \& Piran, T. 1999, ApJ, 517, L109

Wang, X. Y., Dai, Z. G., \& Lu, T. 2000, MNRAS, 319, 1159 